\title{
New equivalent-electrical circuit model and a practical measurement method for human body impedance
}

\author{
Koyu Chinen*, Ichiko Kinjo, Aki Zamami, Kotoyo Irei and Kanako Nagayama \\ Okinawa National College of Technology, 905 Henko, Nago, Okinawa 9052192, Japan
}

\begin{abstract}
Human body impedance analysis is an effective tool to extract electrical information from tissues in the human body. This paper presents a new measurement method of impedance using armpit electrode and a new equivalent circuit model for the human body. The lowest impedance was measured by using an LCR meter and six electrodes including armpit electrodes. The electrical equivalent circuit model for the cell consists of resistance $\mathrm{R}$ and capacitance $\mathrm{C}$. The $\mathrm{R}$ represents electrical resistance of the liquid of the inside and outside of the cell, and the $\mathrm{C}$ represents high frequency conductance of the cell membrane. We propose an equivalent circuit model which consists of five parallel high frequency-passing CR circuits. The proposed equivalent circuit represents alpha distribution in the impedance measured at a lower frequency range due to ion current of the outside of the cell, and beta distribution at a high frequency range due to the cell membrane and the liquid inside cell. The calculated values by using the proposed equivalent circuit model were consistent with the measured values for the human body impedance.
\end{abstract}

Keywords: BIA, human body impedance, equivalent electrical circuit model, armpit electrode

\section{Introduction}

Bio-electrical Impedance Analysis (BIA), utilizing the electrical conductance of ion and cell sap, has been well known as a simplified method to extract electrical information from human body tissues [1-3]. The BIA has been applied to commercial products of body-fat ratio meter that measures the ratio of impedances of the fat and the non-fat tissues [4]. But, the existing measurement method is insufficient in the accuracy of the impedance measurements since it uses high electrical impedance paths through hands, arms and legs $[5,6]$. Especially, the arm has a long part of bone which is electrically nonconductive material. So, it is desirable for the BIA to avoid using the measurement paths of the arms in order to measure the body trunk part which has the lowest impedance in the tissues. Furthermore, we have investigated an equivalent circuit model that represents accurately impedance characteristics of the human body. This report presents a new measurement method for low impedance tissues by using armpit electrodes, and a new equivalent circuit model consisting of parallel RC circuits to represent the human body impedance.

\footnotetext{
* Address for correspondence: Koyu Chinen, Okinawa National College of Technology, 905 Henoko, Nago, Okinawa 9052192, Japan, Tel.: +81980554143; Fax:+81980554012; E-mail: k.chinen@okinawa-ct.ac.jp.
} 


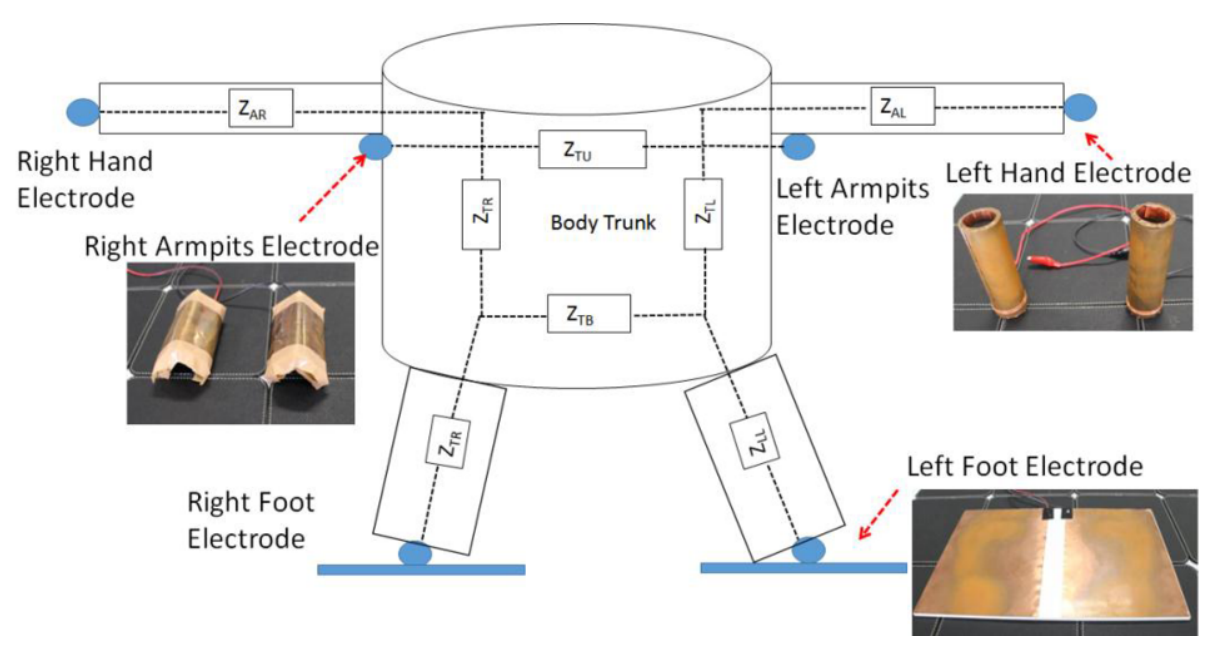

Fig. 1. Human body measurement electrodes with a new electrode for armpits.

\section{Proposal of new measurement method for human body impedances}

\subsection{Use of armpits electrode in measurement for BIA}

We have used a new electrode of armpit, in addition to hand and feet electrodes, as shown in Figure 1. In the lower frequency region than $20 \mathrm{~Hz}$, a large time-constant due to ion current in the tissues was observed during the measurements. In the higher frequency region than $1 \mathrm{MHz}$, an electrical reflectance due to impedance miss-matching was observed. Therefore the proper range of the measurement frequency was from $20 \mathrm{~Hz}$ to $1 \mathrm{MHz}$. A low measurement current between $0.5 \mu \mathrm{A}$ and $70 \mu \mathrm{A}$ was achieved with a low power supply voltage less than $5 \mathrm{mV}$. Since the commercial body-fatratio meter uses a large current of $500 \mu \mathrm{A}$, our measurement current was about one tenth of this value.

The electrode for the hands was made with a polyvinyl chloride tube wrapped with a copper foil. The hardness and the diameter were optimized to fit the strength and size of the grip. The electrode for armpits was made of urethane wrapped with a copper foil. The shape, angle, and elasticity were optimized to fit the armpits. The electrode of the feet was made with a robust plastic plate covered with a copper sheet. The pictures of those electrodes are represented in Figure 1. Since the resistance of the copper material was very low in comparison with the electrical resistance of the skin, the mechanical size of the electrode was not critical in the measurements [7]. Since the change in the skin resistance due to the wet did not affect the impedance measurements at higher frequencies than $20 \mathrm{~Hz}$, the use of the copper electrode was sufficient in the measurements, even with oxidization on the surface during the use for several months. The number of the research participants in the measurements was ten.

\subsection{An LCR measurement system}

Since the human body impedance is widely variable with the measurement frequencies, it was very difficult to accurately measure the impedance by using a function generator and an oscilloscope. The stable and reproductive measurements using a low power supply voltage were realized by using an LCR meter, Agilent 4284A. Figure 2 shows a functional principle of the LCR measurement system. 


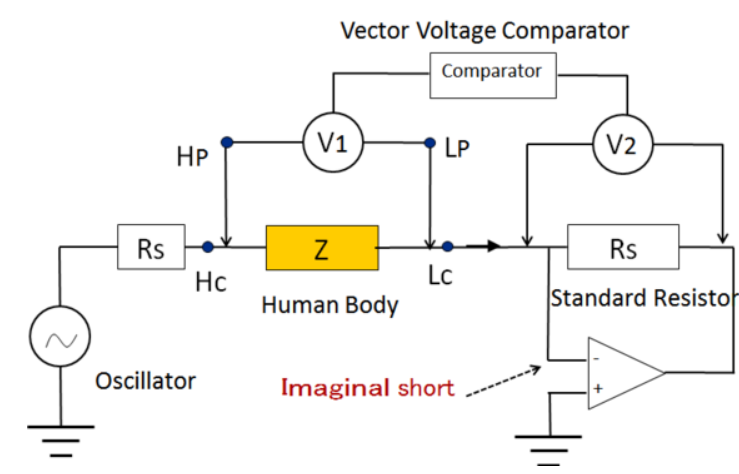

Fig. 2. A functional principle of the LCR meter for stable measurement of the impedance.

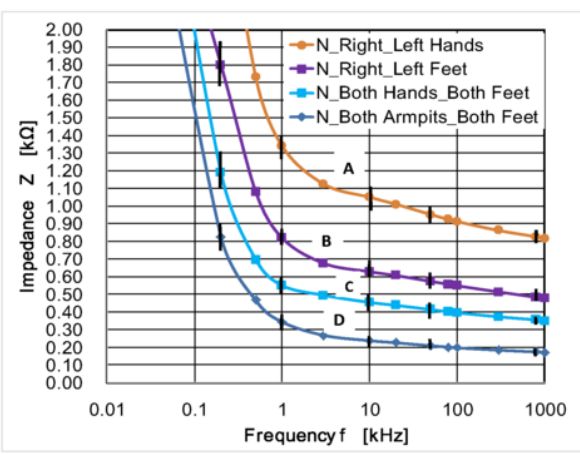

Fig. 3. Human body impedances were measured with various frequencies.

The electrical power generated by an oscillator is applied to the human body and the standard resistor. The difference in the voltage and the phase between the human body and the standard resistor is measured by a vector voltage comparator. Since the one side of the human body was electrically connected to an imaginal ground, the impedance of the human body was correctly and stably measured by using the LCR meter [8]. Since the impedance measurements required different time-constants at low frequencies, we were not able to use any impedance analyzer. In addition, the network analyzer was not suitable for the human body impedance measurement due to the mismatching in the impedance with the 50 -ohms.

\section{Human body impedance measurements results}

A typical impedance measurement result for a human body is shown in Figure 3. The graph shows the relation between the measured frequencies and the impedances. The curve A shows the impedance of the right-left hands electrodes path measurement, and revealed the highest value among the measurements due to the large impedance of the arms. The impedance of the curve A decreases sharply at a frequency around $1 \mathrm{k} \mathrm{Hz}$ due to change of the electrical path in the tissues. The curve B of the left-right feet electrodes path measurement shows about $60 \%$ of the impedance compared to the curve A. Although this measurement method has been used for almost of all fat-ratio meters, using this electrical path was insufficient to measure the lower impedance of the human body trunk. The curve $\mathrm{C}$ of the both hands-both feet electrodes measurement shows relatively low value due to using the parallel conductance. But there was still an influence of high impedance of the arms. The curve D of the both armpits-both legs electrodes measurement shows the lowest value among the measurements. This measurement method was able to avoid the influence of the arms. Since the measurement timeconstant is larger at lower frequencies, the standard error bars show larger error at lower frequencies. According to those measurement results, the impedance measured with armpits electrode most accurately reflects the impedance of the human body trunk that has the lowest impedance.

\section{Proposal of new equivalent circuit model for human body impedance}

\subsection{An equivalent circuit model}




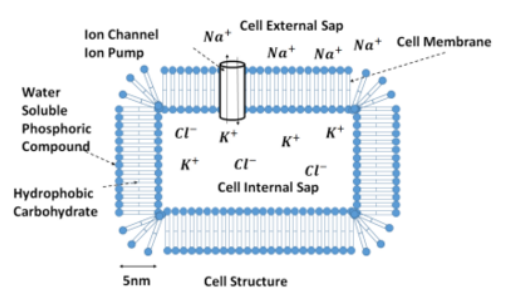

(a)

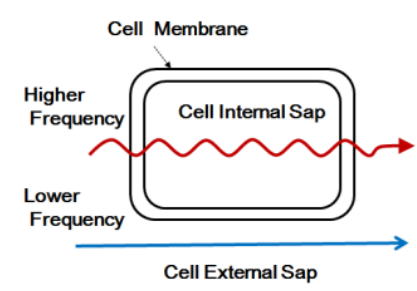

(b)

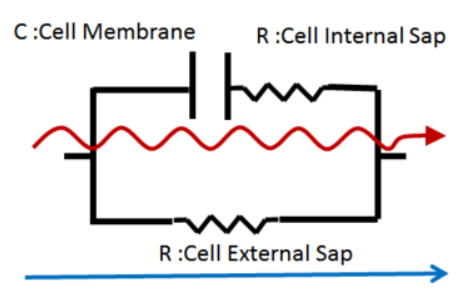

(c)

Fig. 4. (a): cell structure, (b): electrical conductance through the cell, (c):an equivalent circuit.

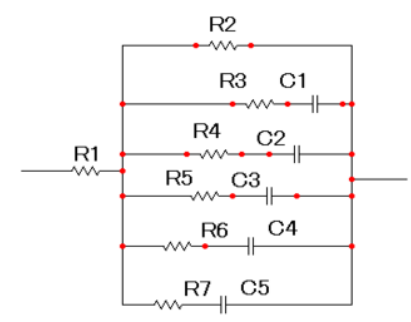

Fig. 5. A new equivalent circuit model with five HPFs for human body impedance.

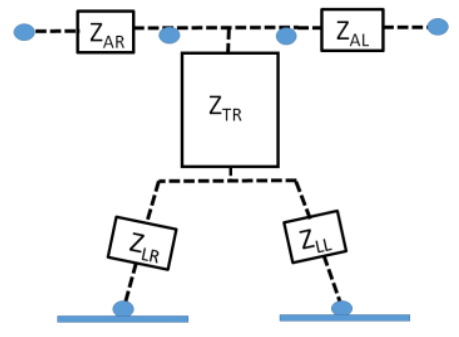

Fig. 6. Impedance of parts of human body extracted from measurements at different frequencies

The human body impedance measurement results suggested an electrical circuit model. The electrical currents with lower frequencies flowing along the outside of the cell, and the currents with higher frequencies flowing along the inside path of the cell are shown in Figure 4(b). A basic electrical circuit model which consists of an equivalent resistor of the sap inside and outside the cell and an equivalent capacitance of the cell membrane is represented in Figure 4(c). But, no detailed electrical circuit model has been reported for the human body due to its complicated organ system. In the experimental measurements, the impedance was sharply decreased at lower frequencies less than about $1 \mathrm{kHz}$ and mostly reached to a low value at higher frequencies than about $10 \mathrm{kHz}$. This phenomenon suggested an equivalent circuit consisting of a combination of $\mathrm{R}-\mathrm{C}$ high pass filters (HPFs). To meet the change in the impedance with frequencies, we propose an equivalent circuit with five HPFs, as shown in Figure 5. The lower number than five was insufficient to meet the measurement results. The higher number than five did not improve the accuracy at the simulation. The simulation results with the proposed equivalent circuit fitted the experimental results measured with fifteen paths in the human body.

In the equivalent circuit model (Figure 6), the electrical admittance of each RC circuit is calculated with the equation of

$$
Y_{n}=\frac{1}{R_{n+2}-j \frac{1}{\omega C_{n}}}
$$

The total admittance of the proposed equivalent circuit is the sum of each admittance.

$$
Y=Y_{1}+Y_{2}+Y_{3}+Y_{4}+Y_{5}
$$


The impedance calculations were carried out with various circuit elements and frequencies, by using Eq. (2).

\subsection{Equivalent circuit models for different measurement paths in the human body}

By using the proposed electrical circuit, several circuit models were created according to various electrical paths in the human body. The values of the circuit elements were determined by using the information from the impedance measurements. In the impedance measurements with the LCR meter, a parallel circuit model and a serial circuit model of the R-C circuits were compared, and the resistance, the phase and the reactance were measured at various frequencies. In case of lower frequencies than $20 \mathrm{~Hz}$, several time-constants for the measurement were used. The applied voltages were changed between $1 \mathrm{mV}$ and $5 \mathrm{mV}$ to reduce the measurement currents and to increase the measurement accuracy. As the combination of the measurement paths, the measurement times at the same day, the dates of the measurements days in a duration of 6 months, and the participants were ${ }_{6} \mathrm{C}_{2}$, 3,4 , and 5 respectively, the total measurements numbers were ${ }_{6} \mathrm{C}_{2} \times 3 \times 4 \times 5$. An example of the list of the values of the equivalent circuit elements of $\mathrm{R}$ and $\mathrm{C}$ that were extracted from those measurements are represented in Table 1. The impedance calculations were carried out based on (2) by using a simulator of Micro-Cap which was a kind of Simulation Program with Integrated Circuit Emphasis (SPICE).

The impedances of five parts in the human body represented in Figure 6 were extracted from the impedance measurements on fifteen paths by using six electrodes, as shown in Table 2 . The impedances of almost all parts were decreased with frequencies, except that of the arm which contained a long length of insulator of the bone. Since the trunk part consists of many electric conductive materials, such as fluid, smooth muscle, and blood vessel, the impedance decreases with frequencies [9]. Various information on the trunk part can be obtained by measuring the impedance between $100 \mathrm{kHz}$ and $800 \mathrm{kHz}$. Although many products for BIA have introduced the arm electrodes to upgrade the performance, as the arms have a large impedance, the use of the arm electrodes should be avoided in the BIA. According to our experiments the arm behaved as an inductor when the frequency was higher than $1 \mathrm{MHz}$. But any material in the human body is not related to the inductor which represents the change in the magnetic flux induced by the change in the applied current.

Table 1

Numerical values of the circuit elements for calculation of impedance for three different electrical paths of a human body

\begin{tabular}{|l|l|l|l|l|l|l|l|l|l|l|l|l|}
\hline Element & $\mathrm{R} 1$ & $\mathrm{R} 2$ & $\mathrm{R} 3$ & $\mathrm{R} 4$ & $\mathrm{R} 5$ & $\mathrm{R} 6$ & $\mathrm{R} 7$ & $\mathrm{C} 1$ & $\mathrm{C} 2$ & $\mathrm{C} 3$ & $\mathrm{C} 4$ & $\mathrm{C} 5$ \\
\hline Unit & $\Omega$ & $\mathrm{k} \Omega$ & $\mathrm{k} \Omega$ & $\mathrm{k} \Omega$ & $\mathrm{k} \Omega$ & $\mathrm{k} \Omega$ & $\mathrm{k} \Omega$ & $\mathrm{nF}$ & $\mathrm{nF}$ & $\mathrm{nF}$ & $\mathrm{nF}$ & $\mu \mathrm{F}$ \\
\hline Path of Right-Left Hands & 150 & 100 & 3 & 8 & 10 & 1.5 & 5 & 1 & 10 & 30 & 150 & 0.3 \\
\hline Path of Right-Right Armpits & 30 & 10 & 1.5 & 8 & 8 & 1 & 1.5 & 1 & 10 & 30 & 80 & 0.3 \\
\hline Path of Both Armpits-Both Feet & 50 & 10 & 0.5 & 1.5 & 1 & 1 & 0.3 & 5 & 10 & 30 & 80 & 1 \\
\hline
\end{tabular}

Table 2

Numerical values of the circuit elements for calculation of impedance for three different electrical paths of a human body

\begin{tabular}{|c|c|c|c|c|c|c|c|c|c|}
\hline \multirow[b]{2}{*}{ Frequency } & \multicolumn{3}{|c|}{$\begin{array}{l}\text { Arm Impedance } \\
\mathrm{Z}_{\mathrm{AR},} \mathrm{Z}_{\mathrm{AL}}\end{array}$} & \multicolumn{3}{|c|}{\begin{tabular}{|l}
$\mathrm{e}$ \\
$\begin{array}{l}\text { Trunk Impedance } \\
\mathrm{Z}_{\mathrm{TRL}}\end{array}$ \\
\end{tabular}} & \multicolumn{3}{|c|}{$\begin{array}{l}\text { Leg Impedance } \\
\mathrm{Z}_{\mathrm{LR},} \mathrm{Z}_{\mathrm{Ll}}\end{array}$} \\
\hline & 10 & 100 & 800 & 10 & 100 & 800 & 10 & 100 & 800 \\
\hline Impedance & 500 & 420 & 420 & 95 & 78 & 50 & 415 & 340 & 287 \\
\hline
\end{tabular}




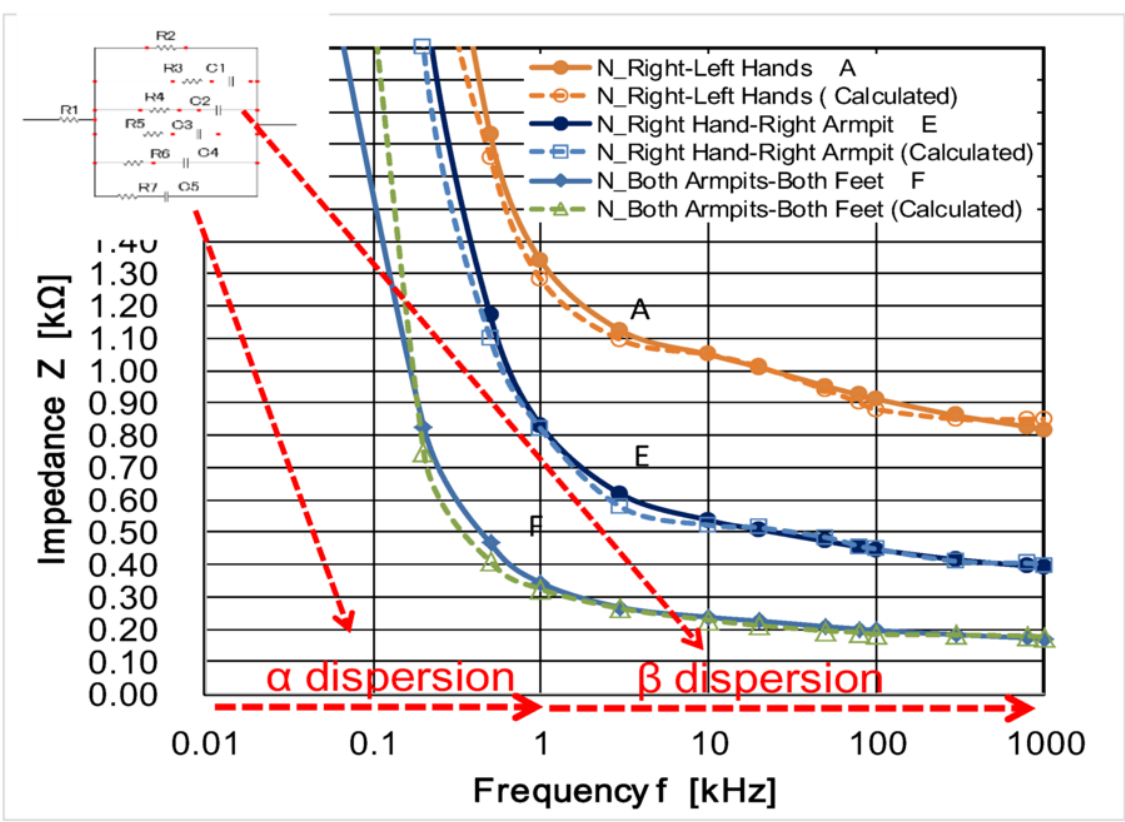

Fig. 7. Comparison between the simulation and the measurement results for human body impedance.

Therefore, the proposed equivalent circuit is valid for measurements with frequencies less than $1 \mathrm{MHz}$.

\section{Comparison of simulation and measurement of impedance}

A comparison of the impedances simulated with the proposed equivalent circuit and the impedances measured with a human body is represented in Figure 7. The measurement values are expressed with solid line curves and the calculated values are expressed with dashed line curves. The impedance measurements were implemented with five participants. Although there were small variations in the shape of the curves, basic features of the curve were similar among the participants. Thus, the typical feature of the impedance curve is represented in Figure 7. The HPFs with large capacitance represent the $\alpha$ dispersion due to ion current at lower frequencies. The HPFs with small capacitance represent the $\beta$ dispersion due to dielectric sap at higher frequencies. The resistor $R$ of each HPF determines the magnitude of the impedance at every frequency. The impedances calculated with the equivalent circuits agreed mostly with the measurement values, as shown in Figure 7. The difference between simulated values and measured values is less than $10 \%$.

\section{Application fields of the proposed circuit model of human body impedance}

In order to create an equivalent electrical circuit for impedance of tissues in the human body, the proposed equivalent circuit can be stacked up as represented in Figure 8. The stacked circuits create a 3D large scale integrated circuit to form various parts of the human body. Although the stacked circuit numbers will be $10 \times 10^{15}$, as the equivalent circuit consists of simplified HPFs, the advanced technologies being developed in simulation and evaluation of the LSI and Nano Scale electronics field 


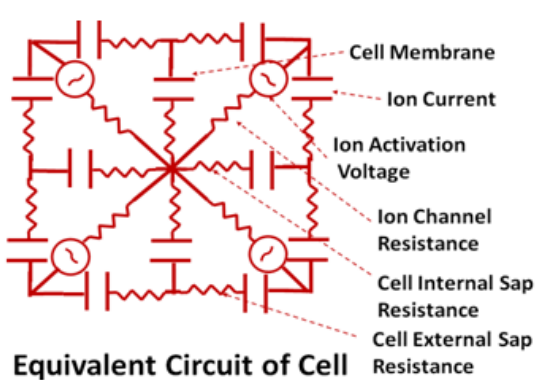

Equivalent Circuit of Cell Resistance

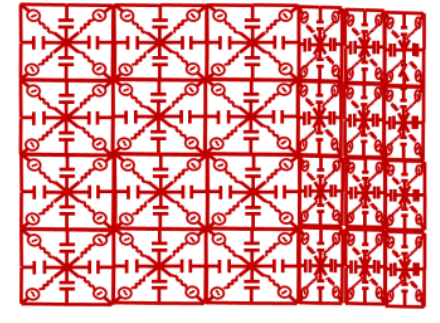

Stacked Equivalent Circuit of Organ

Fig. 8. The equivalent circuit of the cell is stacked to form 3D equivalent circuit for tissues in the human body.

can be used to analyze the electrical characteristics of the complicated and huge stacked circuits. By using the proposed 3D stacked circuits, it will be possible to find electrical solutions to various problems that are confronted in medical treatments, healthy fields, and sick predictions [10-13].

\section{Conclusions}

We have proposed the use of armpit electrodes for measurement of human body impedance. It was confirmed that using both armpit electrodes and both feet electrodes is suitable for measurement of low impedance of the body trunk. The parallel circuit configuration of five HPFs was determined as the equivalent circuit of the human body impedance. The difference between calculated values by the equivalent circuit and the measurement values was less than $10 \%$. The proposed equivalent circuit can be stacked up to create a 3D large scale integrated circuit to form various parts of the human body.

\section{References}

[1] Ursula G. Kylea, et al., Bioelectrical impedance analysis F part I: Review of principles and methods, Clinical Nutrition 23 (2004), 1226-1243.

[2] Bioelectrical Impedance Analysis in Body Composition Measurement, National Institutes of Health Technology Assessment Conference Statement, 1994, pp. 1-35.

[3] F. Ibrahim, M.N. Taib, W.A.B.W. Abas and C.C. Guan, A novel approach to classify risk in dengue hemorrhagic fever (DHF) using bioelectrical impedance analysis (BIA), IEEE Transactions on Instrumentation and Measurement (TIM) 54 (2005), 237-244.

[4] Y. Machii, H. Aoki and K. Koshiji, Fat thickness estimation method using bioelectrical property examination by gel phantom model test, In Proc. 2006 International Symposium on Micro-Nano Mechatronics and Human Science, Nagoya, Japan, 2006, 1-6.

[5] Bioelectrical Impedance Analysis in Estimation of Body Composition, Health Technology Assessment Unit Medical development Division Ministry of Health Malaysia, Oct. 2007, available at: http://www2.moh.gov.my/ attachments/6337

[6] Ching S. Wan, et al., Bioelectrical impedance analysis to estimate body composition, and change in adiposity, inoverweight and obese adolescents: comparison with dual-energy x-ray absorption metry, BMC Pediatrics 14 (2014). doi:10.1186/1471-2431-14-249.

[7] Y. Yamamoto, K. Shirai, N. Goda and T. Nakamura, Evaluating object and region of concentric electrode in bioelectrical impedance measurement, Proceedings of IEEE E in Medicine and Biology Society (EMBS) Asian-Pacific Conference on Biomedical Engineering, Kyoto-Osaka-Nara, Japan, 2003, pp. 282-283.

[8] A.H. Ar-Rawi, M. Moghavvemi and W.M.A. Wan-Ibrahim, Comparing between three current source circuits for using in bio electrical impedance design, Proceeding of 2009 International Conference for Technical Postgraduates (TECHPOS), Kuala Lumpur, Malaysia, Dec.14-15, 2009, 2580-2587.

[9] D. Bracco, D. Thiebaud, R.L. Chiolero, M. Landry, P. Burckhardt and Y. Schutz, Segmental, body composition 
assessed by bioelectrical impedance analysis and DEXA in humans, Journal of Applied Physiology 81 (1996), 25802587.

[10] T. Nakamura and Y. Yamamoto, Evaluation system of physical exercise ability using bio-electrical impedance, In Proc. 2001 IEEE International Symposium on Industrial Electronics, ISIE, Pusan, Korea, Jun12-16, 2001, pp. 2053-2058.

[11] R.C. Kubendran, Sunyoung Kim and R.F. Yazicioglu, Error correction algorithm for high accuracy bio-impedance measurement in wearable healthcare applications, Proceedings of the IEEE International Symposium on Circuits and Systems (ISCAS), Beijing, China, May19-23, 2013, pp. 1292-1295.

[12] Yu-Jie Huang, Yi-Yu Lu and Kuo-Sheng Cheng, Electrical biopsy for radiation enteropathy in rats: Three-element resistance and capacitance model solution of electrical bio-impedance spectroscopy, Proceeding of 4th International Conference on Genetic and Evolutionary Computing, Shenzhen, China, 2010, pp.779-782

[13] K.-S. Kim, Yoon, D.Y. Yang, Y.K. Yang and J.H. Seo, A new bio-impedance sensor technique for leg movement analysis intelligent sensors, Proceedings of the International Conference Series on Intelligent Sensors, Sensor Networks and Information, Melbourne, Australia, 2004, pp. 487-490. 